\title{
5.5 $\mathrm{CO}_{2}$ Cleaning
}

The $\mathrm{CO}_{2}$ method involves spraying $\mathrm{CO}_{2}$ snow against the optical surface. Snow is made using a specialized nozzle (Fig. 5.7) that expands the gas or liquid through an orifice. The snow is propelled at a high velocity, so the impact of the snow strikes any particles or contaminants off the surface. Particles of all sizes, from the visible down to $0.03 \mu \mathrm{m}$, and hydrocarbons are removed quickly. ${ }^{11}$ Figures 5.8 and 5.9 illustrate the effect of this cleaning on an optical surface.

This method is used for critical optics like lasers, telescopes, enclosed, or hard-to-reach optics (within the line of sight from the nozzle to the surface). It may be possible for the snow to reach into grooves and around tubes. $\mathrm{CO}_{2}$ can be used as an interim or the final cleaning step.

The $\mathrm{CO}_{2}$ hardware and the workstation should be in a very clean environment to reduce the chance of re-contamination. Special particle filters such as a HEPA should be used in the cleanroom and/or flow bench. To purge the area and keep the moisture level low, the use of dry air or nitrogen may be required as part of the cleaning. Follow the manufacturer's instructions on the setup and use of the hardware.

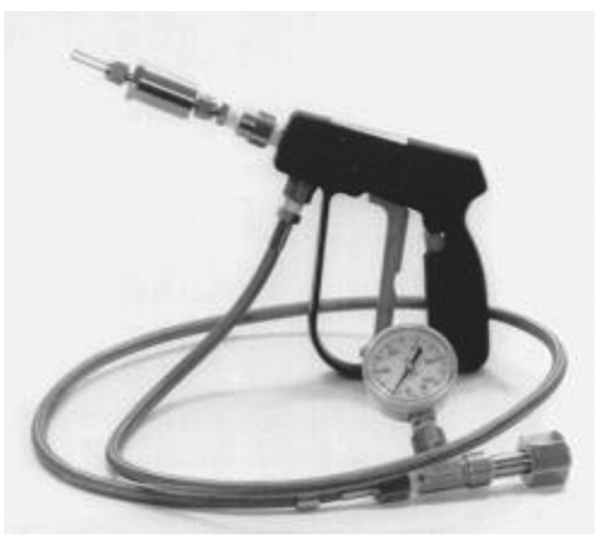

Figure 5.7 $\mathrm{CO}_{2}$ nozzle and control applicator with a pressure gauge (image courtesy of Applied Surface Technologies). 

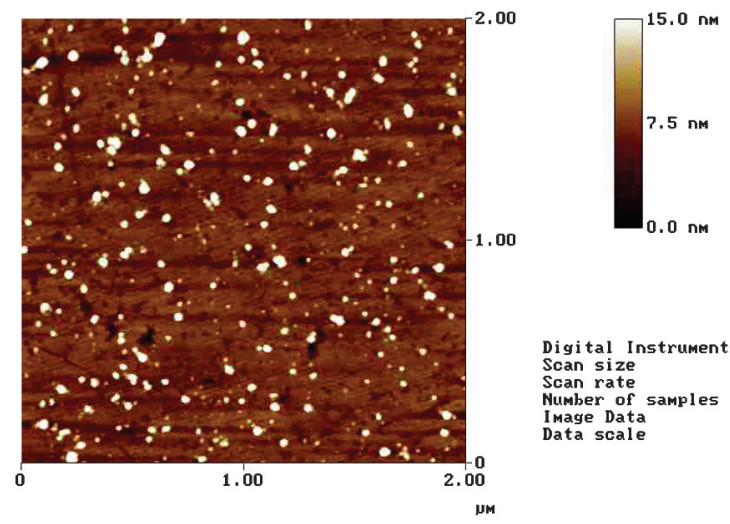

sq1-2um. 101

Figure 5.8 Optical surface before the $\mathrm{CO}_{2}$ cleaning process (image courtesy of Applied Surface Technologies).
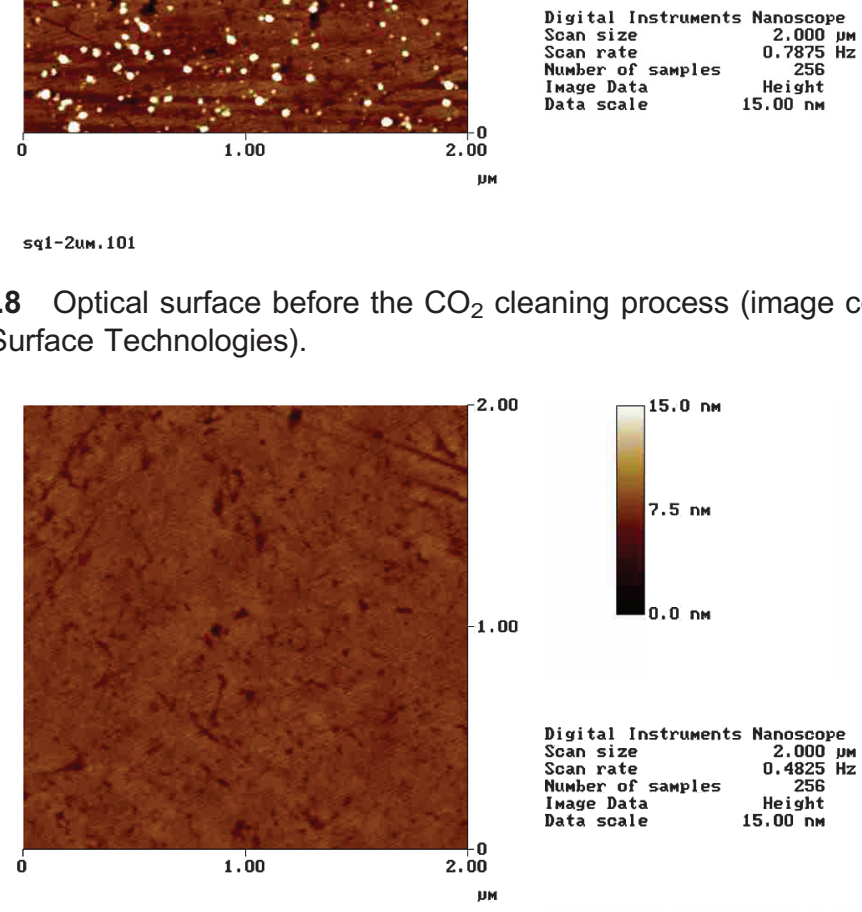

mage Data

Data scale $\quad 15.00 \mathrm{~nm}$

sq1-2um. 102

Figure 5.9 Optical surface after the $\mathrm{CO}_{2}$ cleaning process (image courtesy of Applied Surface Technologies).

The use of a warming plate, heat lamp, warming box, or other warming method is sometimes required to reduce moisture condensation during spraying. A dry box or a nitrogen shroud are alternatives. Caution should be used when applying the cold $\mathrm{CO}_{2}$ to thermally 
sensitive optics or a localized area on very thick glass; be sure to test the procedure on samples.

When using $\mathrm{CO}_{2}$, a static charge build-up is possible and should be addressed.

Observe the effectiveness of the procedure and make adjustments. Select several samples and vary the procedure. On coatings, use the $\mathrm{CO}_{2}$ process cautiously - if the coating has not adhered to the glass, the snow can remove it. An improperly bonded coating can be broken off. ${ }^{9}$

The optic needs to be properly supported at all times and should be secured. The nozzle should be pointed away from the clean parts and the snow should flow away from them. For safety reasons, the area should have good airflow. Do not let the $\mathrm{CO}_{2}$ and nitrogen accumulate in the room or under the flow bench.

\subsection{Ultrasonic Cleaning}

Ultrasonic cleaning uses sound waves to clean optics. The process removes hard-to-clean contaminants from the surfaces and sides of an optic. It can be scaled to handle one optic at a time or hundreds as part of a large cleaning line.

A small hobby ultrasonic cleaner can be used for experimental cleaning; they are typically used for small items such as jewelry. A cleaner of this size can handle optics up to $50-60 \mathrm{~mm}$. As a precaution, test a few samples of the type of glass to be cleaned and observe the effect of the ultrasonic process on the glass. The mixture of cleaning liquids and the agitation of the ultrasonic waves can have adverse effects. Although benign when used alone, combining sound waves and cleaning liquids can damage an optic.

Start by pouring a simple soap-and-water mixture into an ultrasonic tub. This mixture is used to clean polishing compounds off of the edges of an optic, and oils and fingerprints from the surfaces. The percentage of soap to water should be about $1 \%$ of the volume of water. 
A simple plastic holder or rack is recommended to place the optic in the cleaner; it should have an open design to allow for proper cleaning. The optic should be held on its edge or laid flat, and secured to prevent movement during cleaning (in such a way that the optical surface does not touch the insides or bottom of the cleaning chamber). This precaution prevents vibrations from causing subsurface damage to the optical surface. Leave the optic in the cleaner and bath for a limited amount of time: the process should be monitored every 30-90 s.

\subsection{Carbon-Tip Cleaning}

This method uses a fine carbon powder that is embedded in felt or chamois material. The cleaning process is very similar to the chalk method, where a fine powder scrubs and absorbs oils and other contaminants. It works best on uncoated optical surfaces or coated surfaces with hard AR coatings. Carbon tips have the advantage of removing oils and stains without the use of liquids, and the felt or chamois softness reduces the risk of scratching the optical coating or surface. A carbon wipe can be used on flat surfaces, such as tablets, screens (e.g., computers and smartphones; see Fig. 5.10), and windows.

Do not use carbon tips on soft coatings such as unprotected front-surfacemirror coatings. If in doubt, test on a sample first. Review and follow the manufacturer's instructions on its use and limitations. Also, do not use carbon tips on optical surfaces that have large amounts of moisture, dirt, or oils, as they will be less effective. Moisture and oils will be absorbed into the felt, chamois tip, or wipe.

The carbon-tip tool has a fine brush attached on the opposite end of the tool; the brush is used first to remove large dust particles (see Fig. 5.11). When finished, remove the cap covering the carbon tip (note that it contains a small amount of carbon powder to replenish the tip). The tip is then lightly pressed against the optical surface and slowly moved in a spiral motion from the center out to the edge (Fig. 5.12). During the final rotation, lift the tip up and away from the surface. 


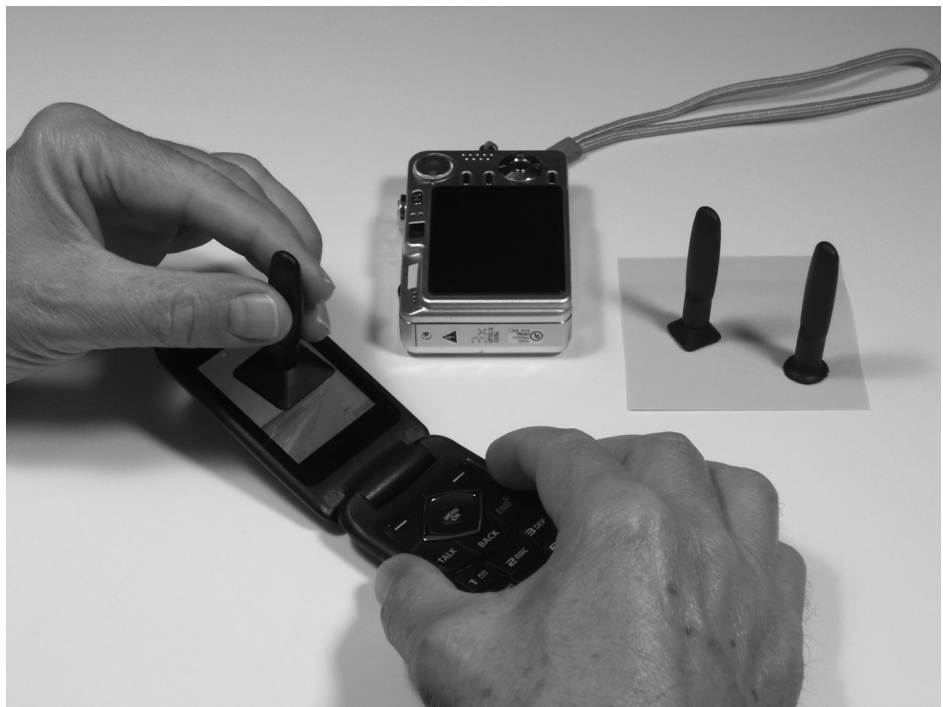

Figure 5.10 Cleaning the surface of a cell phone screen using a carbon-tip wipe.

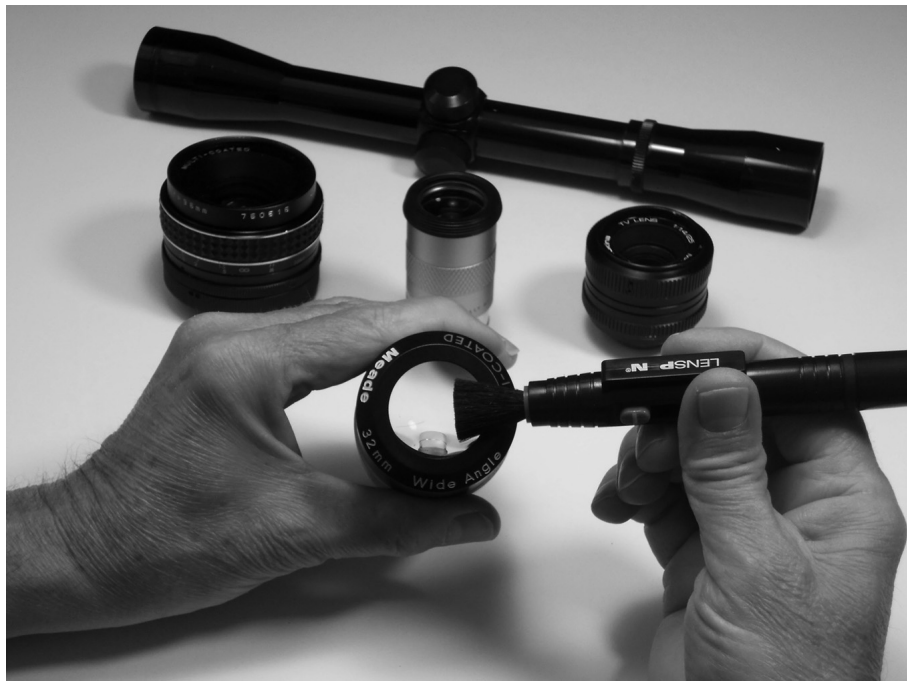

Figure 5.11 Cleaning the eye lens of an eyepiece with a small brush before using the carbon tip. 